\title{
Gastrocutaneous Fistula in a Patient with Locally Recurrent MSI-High Colorectal Cancer: Local Complications Arising from Therapeutic Response to Immune Checkpoint Blockade
}

\author{
CLAIRE GREENE ${ }^{1}$, ERIC K. NAKAKURA ${ }^{2}$ and ANDREW H. KO ${ }^{1}$ \\ ${ }^{1}$ Division of Hematology/Oncology, ${ }^{2}$ Division of Surgical Oncology, \\ Helen Diller Family Comprehensive Cancer Center, University of California, San Francisco, CA, U.S.A.
}

\begin{abstract}
Colorectal cancers with high microsatellite instability (MSI-H) have distinct clinical features in terms of their prognosis, recurrence patterns, and sensitivity to immunotherapeutic agents. We present the case of a woman with a left-sided MSI-H colon cancer who had repeated recurrences concentrated exclusively in the left upper quadrant of the abdomen, including gastric involvement. Despite multiple surgical resections, radiation, and several lines of chemotherapy, her disease eventually eroded through the chest wall. Treatment with an immune checkpoint inhibitor produced a rapid clinical response with significant tumor necrosis; however, this necessitated surgical debridement that ultimately led to a large gastrocutaneous fistula. This case highlights the importance of recognizing locoregional tumor-associated complications that may result from robust therapeutic responses to immuno-oncology drugs, which are increasingly being used in clinical practice today.

Approximately $15 \%$ of colorectal cancers exhibit high levels of microsatellite instability (referred to as MSI-H), indicating a clonal change in the number of repeated DNA nucleotide units in stretches of DNA sequence (termed microsatellites) that occur throughout the genome (1). This MSI-H phenotype arises as a result of an intrinsic deficiency in DNA mismatch repair (MMR) due to the inactivation of one or more MMR genes (MLH1, MSH2, MSH6, and PMS2), thus leading to the nomenclature of dMMR (as opposed to proficient or pMMR)
\end{abstract}

This article is freely accessible online.

Correspondence to: Dr. Andrew H. Ko, Division of Hematology/ Oncology, University of California, 550 16th Street, 6th floor, Box 3211, San Francisco, CA 94143. Tel: +1 4153537286, e-mail: andrew.ko@ucsf.edu

Key Words: Immunotherapy, PD-1, fistula, microsatellite instability, mismatch repair deficiency. tumors. While there is a strong association of MSI-H/dMMR colorectal tumors with Lynch Syndrome, also known as Hereditary Non-Polyposis Colorectal Cancer (HNPCC), this molecular subtype can also be seen with sporadic forms of colorectal cancer, typically via epigenetic silencing of MLH1.

The therapeutic implications of MSI-H colorectal cancers are being increasingly recognized. Clinical studies dating back more than a decade have suggested that patients with MSI-H stage II or III colorectal tumors do not appear to derive any benefit from fluoropyrimidine-based adjuvant chemotherapy $(2,3)$. More recently, data from immunotherapy-based clinical trials have indicated the benefits of treatment with an anti-programmed death 1 (PD-1) immune checkpoint inhibitor appears to be limited primarily to colorectal cancers that are MSI-H, with minimal to no clinical activity in those that are microsatellite stable (MSS) (4). In such MSI-H patients, the depth and duration of responses to such immune-based treatments can be quite striking, offering the promise to move the field beyond traditional cytotoxic and VEGF/EGFR-targeted agents.

While the side-effect profiles of anti-PD-1 monoclonal antibodies have been very well characterized at this point, particularly with regard to immune-related adverse events that may affect almost any part of the body, the potential for local complications occurring at specific tumor locations as a result of robust treatment response remains underappreciated. We present the case of a woman with an MSI-H colon cancer with multiple bulky tumor recurrences involving the chest wall who responded dramatically to the PD-1 inhibitor pembrolizumab. However, this tumor response ultimately required tissue debridement and resulted in a persistent nonhealing gastro-cutaneous fistula.

\section{Case Presentation}

S.B. was a previously healthy 49-year-old woman who originally presented in July 2012 with several months of low-grade fevers, chills, and fatigue. Laboratory evaluation indicated profound anemia, with a hemoglobin of $6 \mathrm{~g} / \mathrm{dl}$, 
prompting a colonoscopy that revealed a friable, ulcerated, circumferential mass in the descending colon located from 35 to $55 \mathrm{~cm}$ from the anal verge. CT scan confirmed a large mass at the splenic flexure eroding into the gastrosplenic ligament.

The patient was taken for surgery, where intraoperatively she was found to have a large tumor at the splenic flexure of the colon eroding into the splenic hilum and distal pancreas, with a contained perforation posterior to the spleen. Surgery consisted of en bloc tumor resection including left hemicolectomy, splenectomy, and distal pancreatectomy. The spleen itself was quite adherent to the diaphragm and lateral abdominal wall; consequently, dissection resulted in diaphragmatic injury and a small rent in the pleura requiring repair. Final pathology showed an $8 \mathrm{~cm}$, high-grade adenocarcinoma with focal mucin secretion, invasive through the full thickness of the muscularis into the subserosal fat and perisplenic and peripancreatic fibrous tissue (T4b). No carcinoma was found in 10 evaluable lymph nodes, but 4 satellite nodules (tumor deposits) were identified in the mesenteric fat (N1c). Surgical margins were negative. Molecular testing revealed a KRAS mutation (codon not specified on laboratory report), as well as evidence of microsatellite instability using a multiplex PCR reaction amplifying five mononucleotide repeat markers. By immunohistochemistry, however, all 4 mismatch repair proteins (MLH1, MSH2, MSH6 and PMS2) showed intact nuclear expression.

The patient's medical history was otherwise unremarkable, with no chronic medical conditions or prior surgeries. She was a non-smoker and did not drink alcohol. Her family history was notable for a maternal aunt with breast cancer in her 50s, a paternal great grandmother with colon cancer in her $90 \mathrm{~s}$, and a paternal second cousin with colon cancer in his 50s. She underwent germline testing using the ColoNext genetic testing panel at Ambry Genetics, which did not reveal any deleterious mutations. Of the MMR genes, only a variant of unknown significance in PMS2 (p.G108R) was noted.

Following postoperative recovery, the patient received 12 cycles of adjuvant FOLFOX (5-fluorouracil, leucovorin, oxaliplatin). CT-PET scan performed at the completion of adjuvant therapy showed hypermetabolic activity in the left upper quadrant extending to the pancreatectomy bed measuring $34 \times 29 \mathrm{~mm}$, concerning for locally recurrent disease. On this basis, she received radiation therapy $(5,040 \mathrm{cGy})$ to this area with concurrent capecitabine from March to April 2013. Follow-up CT-PET 3 months later continued to show hypermetabolic activity in this same area, slightly decreased in size but with increased FDG uptake. The patient was then started on FOLFIRI combined with bevacizumab, which she received for a total of 12 cycles. While follow-up imaging shortly after completion of treatment showed no residual measurable disease, repeat CT 3 months later showed the appearance of a $4.1-\mathrm{cm}$ lobular mass along the posterior wall of the stomach, compatible with local recurrence.

She was taken back to the operating room in May 2014 (22 months from the time of her original operation), where intraoperatively the mass was noted to be adherent to the posterior aspect of the stomach in the fundus, tethered to the diaphragm and to the antimesenteric portion of the colon. Surgery required en bloc distal pancreatectomy, partial colectomy, partial gastrectomy, and partial diaphragm resection, plus intraoperative radiotherapy (IORT) to a total of 15 Gy. Final pathology revealed residual/recurrent adenocarcinoma with extensive necrosis, $4.2 \mathrm{~cm}$ in maximum dimension, invading through the stomach wall into the gastric antral mucosa and the soft tissue deep to diaphragm. Margins were negative and there was no tumor involvement of adjacent organs.

Follow-up CT scan 4 months postoperatively again showed recurrent disease along the greater curvature of her stomach along with a couple of adjacent LUQ soft tissue nodules, for which she was restarted of FOLFIRI for a total of 13 cycles, with the addition of afliberacept for the last 6 . She responded well to this regimen with smaller but persistent disease on repeat imaging, at which point she was taken again to surgery consisting of a left thoracoabdominal resection, partial gastrectomy and colectomy, left diaphragm and chest wall resection and reconstruction, and left adrenalectomy. Final pathology revealed only areas of necrotic debris and inflammation, with no viable tumor.

Repeat CT-PET 4 months following this operation showed a growing hypermetabolic area along the $\mathrm{L}$ posterior inferior chest wall, near the $\mathrm{L} 11$ th rib resection site, prompting the patient to resume chemotherapy with FOLFIRI/afliberacept. However, her disease failed to respond this time around. She was then tried on abbreviated courses of regorafenib (Stivarga) and TAS-102 (Lonsurf) were poorly tolerated and lasted less than one month apiece. Treatment was then interrupted when she developed a left-sided thoracic abscess/empyema. Attempted percutaneous drainage was unsuccessful, and she was treated with a 4-week course of intravenous vancomycin and ertapenem.

Thereafter, the patient enrolled on a clinical trial evaluating pembrolizumab for subjects with previously treated locally advanced unresectable or metastatic dMMR/MSI-H colorectal cancers (KEYNOTE 164; NCT 02494583). Study treatment was initiated in June 2016, at which time her malignant-appearing mass was fungating significantly through the left posterolateral chest wall (Figures 1A and 2).

Within the first two cycles of pembrolizumab, administered on a 3-week basis, the visible chest wall lesion began to show evidence of significant softening and necrosis, with sloughing of dead tumor tissue and oozing of 

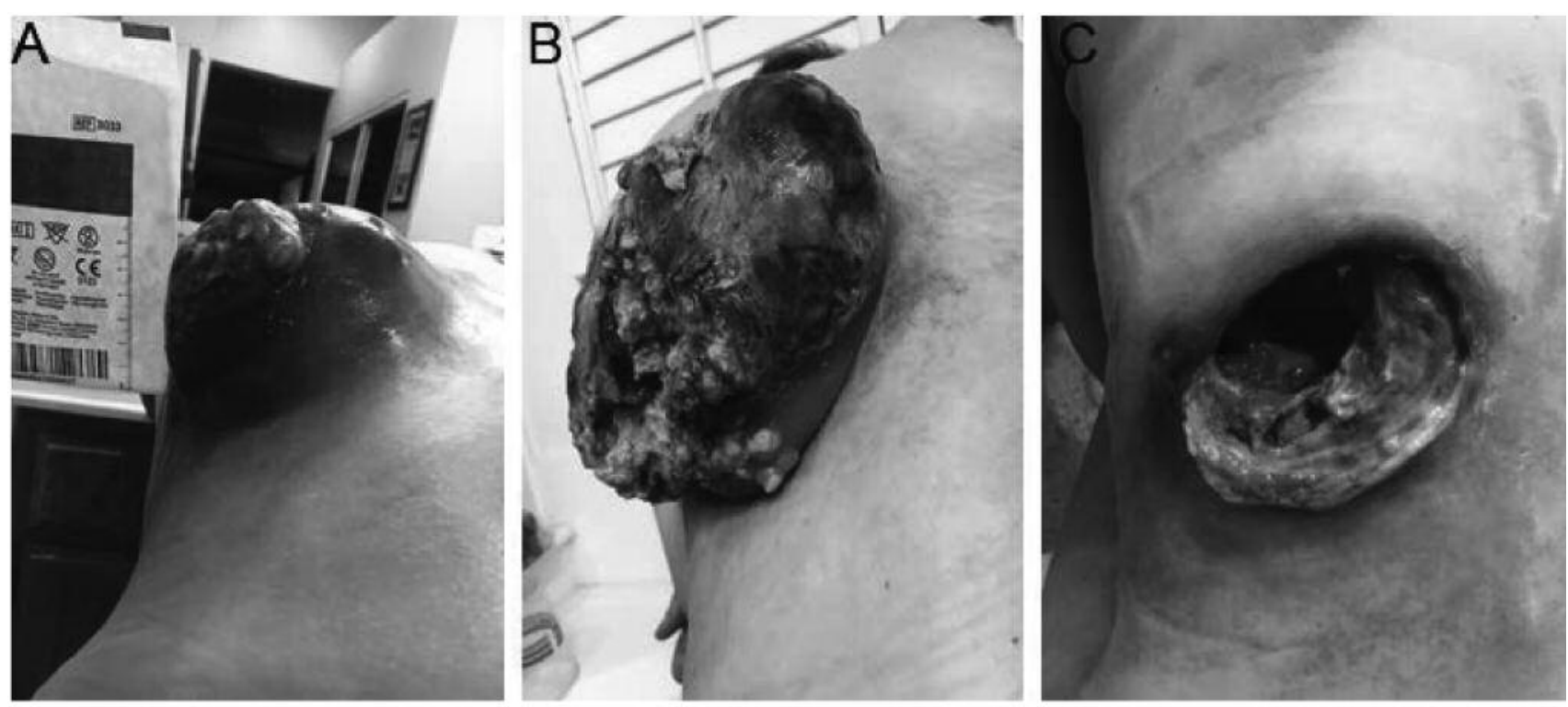

Figure 1. (A) Fungating chest wall mass prior to initiation of pembrolizumab. (B) Evidence of tumor necrosis following 2 cycles of treatment. (C) Chest wall cavity following tumor debridement, with gastrocutaneous fistula.

malodorous brown, dark-colored fluid with some particulate matter (Figure 1B). Surgery was consulted and performed surgical debridement of this malignant tumor mass (as an outpatient procedure) for hygienic and cosmetic purposes.

Beginning the following day, the patient reported significant drainage from the chest wall cavity (Figure 1C), consisting of copious amounts of fluid mixed with partially digested food and stomach contents. She was immediately admitted to the hospital for management of this gastrocutaneous fistula, with placement of a nasojejunal tube for initiation of tube feeds, instructions on optimal wound care at home, including topical application of an ostomy bag over the chest wall wound, and packed red blood cell transfusion for a hemoglobin that nadired at $7.1 \mathrm{~g} / \mathrm{dl}$.

Despite this complication, the patient was judged to have achieved a favorable treatment response, and following discharge was resumed on pembrolizumab per study protocol. She received an additional 5 treatment cycles (7 in total), during which time her fistula showed some evidence of partial closure although it continued to produce some drainage of varying amount, color, and consistency. She remained entirely dependent on nasojejunal tube feeds this entire time, with her p.o. intake limited to ice chips and small sips of water. However, during the latter course of her treatment, the patient began experiencing intermittent lowgrade fevers, with one positive anaerobic blood culture for Fusobacterium nucleatum, and was noted on serial laboratory measurements to be developing progressively worsening cytopenias. She was rehospitalized in October

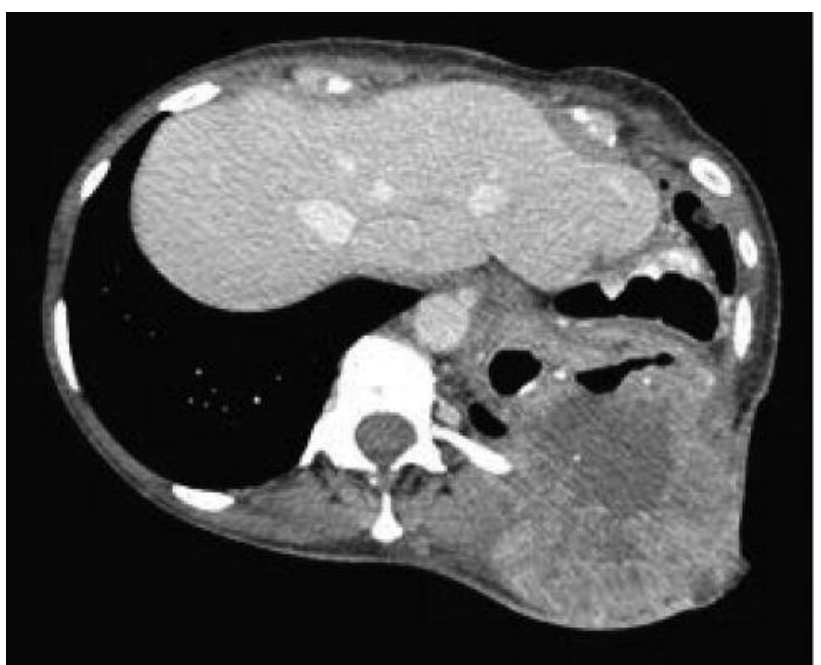

Figure 2. Cross-sectional imaging (CT scan with contrast) prior to start of pembrolizumab demonstrating large fungating mass centered in the left upper quadrant, lung base, and pleural space, in direct communication with the stomach and eroding through the left posterior chest wall.

2016 for progressive fatigue, shortness of breath, and failure to thrive, at which point her hemoglobin was $6.4 \mathrm{~g} / \mathrm{dL}$ and her platelet count $27 \times 10^{9} / \mathrm{L}$, representing a decrease from $328 \times 10^{9} / \mathrm{L}$ less than two months earlier. Flow cytometry performed on a peripheral blood sample showed acute myeloid leukemia with $60 \%$ blasts expressing variable CD7, 
CD13, CD33, CD34, CD38, weak CD64, weak CD71, weak CD123, and HLA-DR without co-expression of MPO, TdT, or lymphoid antigens (other than CD7). She was started on palliative treatment with hydroxyurea, but her clinical condition worsened with progressive respiratory distress due to pleural and pericardial effusions of indeterminate etiology, along with a rising white blood cell and blast count into the $50 \mathrm{~s}$. The decision was made to transition to comfort care, and she ultimately died one week after hospitalization.

\section{Discussion}

Patients with localized MSI-H/dMMR colorectal cancers typically have a favorable prognosis, with relatively low rates of recurrence following surgical resection of the primary tumor. As a result, only a small number of studies have been able to examine patterns of relapse and clinical outcomes in such individuals. Sinicrope and colleagues performed a retrospective analysis of 2,141 patients with resected stage II and III colon cancers participating in United States cooperative group adjuvant trials, reporting reduced rates of tumor recurrence, delayed time to recurrence, and improved stage- and treatment-adjusted disease-free and overall survival for dMMR compared to pMMR tumors (1). A more recent study reported by Kim et al. of 2,940 Korean patients with resected stage I-III colorectal cancer also found different recurrence patterns between MSI-H and MSIlow/MSS patients, with the former group more frequently demonstrating local recurrence or peritoneal metastases, with a correspondingly lower rate of either lung or liver metastases (5).

It is important to recognize that the patient presented here had very locally advanced (T4) disease at original presentation, placing her at an extremely high risk of locoregional recurrence regardless of MSI status. However, her pattern of multiple recurrences located exclusively in the left upper quadrant/chest wall without development of other sites of distant metastases was somewhat atypical. This relapse pattern did afford her the opportunity to receive a variety of both systemic and local treatments, including multiple surgeries and radiation, throughout her disease course. Ultimately, after exhausting her standard chemotherapeutic options, she was enrolled onto an immunotherapy-based clinical trial for which she was eligible based on the MSI-H status of her tumor.

Multiple medical groups, including the U.S. Multi-Society Task Force on Colorectal Cancer, now recommend universal testing of MMR deficiency in all patients with newly diagnosed colorectal cancer, based on its prognostic and therapeutic implications (6). Analysis can be performed by immunohistochemical testing for MMR proteins and/or testing for MSI by a polymerase chain reaction (PCR)-based assay. Our patient was confirmed to be MSI-H by PCR in 5 of 5 microsatellite loci tested, but interestingly, IHC staining demonstrated intact expression of MLH1, MSH2, MSH6, and PMS2 proteins. While strong correlation is reported between MSI DNA analysis and IHC for determining MMR status $(7,8)$, it is possible on occasion to see discordant results due to technical issues in slide fixation, observer error, or rare cases in which missense mutations in MLH1 or MSH6 result in a normally translated but non-functional protein (9). Notably, germline testing in this patient failed to detect mutations in any of the 4 MMR genes.

Microsatellite instability appears to represent a useful biomarker in colorectal cancer and other tumors for predicting sensitivity to immune checkpoint blockade, as it leads to a hypermutated phenotype in which a large number of neoantigens are generated that may stimulate an immune response (10). In a seminal phase II study reported by Le $e t$ al., objective responses to the anti-PD1 monoclonal antibody pembrolizumab were observed in $40 \%$ (4/10) of patients with dMMR metastatic colorectal cancers, compared to $0 \%(0 / 18)$ of patients with pMMR tumors (4). Updated results from this same study with additional patients $(\mathrm{n}=28)$ and more mature data indicated an even more robust response rate $(57 \%)$ in the dMMR subgroup (11). A subsequent phase II trial presented in abstract form by Overman and colleagues evaluated another anti-PD-1 antibody, nivolumab, either alone or in combination with the cytotoxic T-lymphocyteassociated protein 4 (CTLA-4) antibody ipilimumab, in patients with dMMR metastatic colorectal cancer (12). Interim analysis from the nivolumab monotherapy cohort showed a response rate of $31 \%$ (23/74), with a number of durable responses lasting more than one year. On these bases, immunotherapy has been added to the treatment guidelines of the National Comprehensive Cancer Network (NCCN) for patients with advanced or metastastic dMMR/MSI-H colorectal cancers who have progressed on standard chemotherapy (13), while the U.S. Food and Drug Administration approved pembrolizumab in May 2017 for previously treated patients with advanced MSI-H cancers including colorectal.

Given the favorable toxicity profile associated with immune checkpoint blockade, our patient was deemed an appropriate clinical trial candidate for pembrolizumab-based study treatment despite her moderate frailty and fungating chest wall mass. Skin metastases may be seen in upwards of $5 \%$ of patients with colorectal cancer; however, cutaneous involvement by direct tumor extension is uncommon (14-16). This patient's multiple prior debulking surgical procedures plus radiation, all concentrated in a single anatomic area, may have contributed to chronic inflammation and tissue friability that increased the likelihood of her recurrent tumor invading into and eventually breaking through the skin. At the point of initiating immunotherapy, CT imaging showed her tumor mass to be communicating directly between the stomach wall and 
the skin and muscle layers of the left chest wall, raising the distinct possibility that a favorable response to treatment could directly or indirectly lead to fistulous tract formation. Indeed, gastric perforation induced by treatment has been described for various tumor types, most notably Burkitt's lymphoma given its marked chemosensitivity (17); but also primary gastric cancer (18) and metastasis to the stomach (19).

To our knowledge, this is the first reported case of tumor necrosis induced by immunotherapy leading to gastrocutaneous fistula formation, with the fistulous output prompted or exacerbated by surgical debridement that was considered a necessary component in the patient's clinical care. While it is critical to recognize the risks associated with treating locally advanced and full-thickness tumors that extend through the wall of luminal organs, including perforation, rupture, and fistula formation, the challenge lies in trying to enact preemptive measures to minimize or prevent such complications. Provider awareness as well as counseling of patients to be vigilant of signs or symptoms of any of the above complications are critical for early detection and rapid management before they become life-threatening. Once this patient's gastrocutaneous fistula was recognized, she was immediately hospitalized and provided supplemental enteral nutrition via nasojejunal tube feeding, with explicit instructions to her and her caregiver regarding appropriate wound care and hygiene given the high risk for infection. Management of fistulae of this sort can range from conservative medical management to endoscopic placement of glue, clips, or stent to surgical repair, depending on factors including the size and severity of the fistula and the patient's overall clinical condition (20). Spontaneous closure is relatively uncommon. In this patient's case, a more aggressive interventional approach for her fistula was felt to be contraindicated, with efforts made instead to improve her nutritional status, encourage optimal wound management, and allow her to continue with her cancer immunotherapy in the process.

Gradually, the patient's fistula did show signs of healing, albeit not completely. Her ultimate demise 4 months later was unrelated to this persistent fistula, but instead due to the unexpected development of acute myeloid leukemia (AML). An analysis of data from the Surveillance, Epidemiology, and End Results (SEER) Program between 1975-2001 indicated that colorectal carcinoma patients do have significantly higher incidences of certain secondary malignancies, including small intestine, stomach, kidney, and endometrial cancers, presumably reflecting hereditary cancer conditions such as Lynch Syndrome (21). Hematologic malignancies, meanwhile, are not recognized as occurring at a higher rate in the colorectal cancer survivor population. Another possibility to consider in this patient's case was whether she developed a therapy-related myeloid neoplasm, an entity that includes both myelo- dysplastic syndrome (MDS) and AML and occurs at a median of 3-5 years post-treatment (22). However, therapyrelated ( $\mathrm{t}-$ )AML typically develops as a result of either alkylator or topoisomerase II exposure (23), neither class of agent which this patient received, although it is conceivable that her prior radiation may have played some role. There is no evidence to support her ongoing immune checkpoint therapy as a contributing factor to her leukemia. In retrospect, diagnostic work-up of her progressive cytopenias may have been undertaken earlier, although it is unlikely that an earlier diagnosis of AML would have significantly impacted on her care and prognosis. Decreased blood counts represent a rare side effect of PD-1 antibody therapy, although both immune-related hemolytic anemia and thrombocytopenia have been reported $(24,25)$.

In summary, we highlight this patient's case based on a number of instructive and unusual aspects: the atypical pattern of multiple local recurrences of an MSI-H colorectal cancer, the exquisite response to immune checkpoint inhibitor therapy with resultant development of a gastrocutaneous fistula, and the late occurrence of a hematologic malignancy of unclear relation to earlier events. As perhaps the most valuable take-home lesson, we submit that with immunotherapy now ushering in a new paradigm for treating patients with a variety of solid tumors, it becomes important to recognize the potential for locoregional complications that may ensue, even if they may be considered, in some sense, a byproduct of therapeutic success.

\section{Acknowledgements}

The Authors would like to express deep appreciation to the patient's next of kin for giving permission to publish this case report and all accompanying images.

\section{References}

1 Sinicrope FA, Foster NR, Thibodeau SN, Marsoni S, Monges G, Labianca R, Kim GP, Yothers G, Allegra C, Moore MJ, Gallinger S and Sargent DJ: DNA mismatch repair status and colon cancer recurrence and survival in clinical trials of 5fluorouracil-based adjuvant therapy. J Natl Cancer Inst 103: 863$875,2011$.

2 Ribic CM, Sargent DJ, Moore MJ, Thibodeau SN, French AJ, Goldberg RM, Hamilton SR, Laurent-Puig P, Gryfe R, Shepherd LE, Tu D, Redston M and Gallinger S: Tumor microsatelliteinstability status as a predictor of benefit from fluorouracil-based adjuvant chemotherapy for colon cancer. N Engl J Med 349: 247-257, 2003.

3 Sargent DJ, Marsoni S, Monges G, Thibodeau SN, Labianca R, Hamilton SR, French AJ, Kabat B, Foster NR, Torri V, Ribic C, Grothey A, Moore M, Zaniboni A, Seitz JF, Sinicrope F and Gallinger S: Defective mismatch repair as a predictive marker for lack of efficacy of fluorouracil-based adjuvant therapy in colon cancer. J Clin Oncol 28: 3219-3226, 2010. 
4 Waddell N, Pajic M, Patch AM, Chang DK, Kassahn KS, Bailey P, Johns AL, Miller D, Nones K, Quek K, Quinn MC, Robertson AJ, Fadlullah MZ, Bruxner TJ, Christ AN, Harliwong I, Idrisoglu S, Manning S, Nourse C, Nourbakhsh E, Wani S, Wilson PJ, Markham E, Cloonan N, Anderson MJ, Fink JL, Holmes O, Kazakoff SH, Leonard C, Newell F, Poudel B, Song $\mathrm{S}$, Taylor D, Waddell N, Wood S, Xu Q, Wu J, Pinese M, Cowley MJ, Lee HC, Jones MD, Nagrial AM, Humphris J, Chantrill LA, Chin V, Steinmann AM, Mawson A, Humphrey ES, Colvin EK, Chou A, Scarlett CJ, Pinho AV, Giry-Laterriere M, Rooman I, Samra JS, Kench JG, Pettitt JA, Merrett ND, Toon C, Epari K, Nguyen NQ, Barbour A, Zeps N, Jamieson NB, Graham JS, Niclou SP, Bjerkvig R, Grutzmann R, Aust D, Hruban RH, Maitra A, Iacobuzio-Donahue CA, Wolfgang CL, Morgan RA, Lawlor RT, Corbo V, Bassi C, Falconi M, Zamboni G, Tortora G, Tempero MA, Gill AJ, Eshleman JR, Pilarsky C, Scarpa A, Musgrove EA, Pearson JV, Biankin AV and Grimmond SM: Whole genomes redefine the mutational landscape of pancreatic cancer. Nature 518: 495-501, 2015.

5 Kim CG, Ahn JB, Jung M, Beom SH, Kim C, Kim JH, Heo SJ, Park HS, Kim JH, Kim NK, Min BS, Kim H, Koom WS and Shin SJ: Effects of microsatellite instability on recurrence patterns and outcomes in colorectal cancers. Br J Cancer 115: 25-33, 2016.

6 Giardiello FM, Allen JI, Axilbund JE, Boland CR, Burke CA, Burt RW, Church JM, Dominitz JA, Johnson DA, Kaltenbach T, Levin TR, Lieberman DA, Robertson DJ, Syngal S and Rex DK: Guidelines on genetic evaluation and management of Lynch syndrome: a consensus statement by the US Multi-Society Task Force on colorectal cancer. Gastroenterology 147: 502-526, 2014.

7 Lindor NM, Burgart LJ, Leontovich O, Goldberg RM, Cunningham JM, Sargent DJ, Walsh-Vockley C, Petersen GM, Walsh MD, Leggett BA, Young JP, Barker MA, Jass JR, Hopper J, Gallinger S, Bapat B, Redston $M$ and Thibodeau SN: Immunohistochemistry versus microsatellite instability testing in phenotyping colorectal tumors. J Clin Oncol 20: 1043-1048, 2002.

8 Chen W, Swanson BJ and Frankel WL: Molecular genetics of microsatellite-unstable colorectal cancer for pathologists. Diagn Pathol 12: 24, 2017.

9 Zhang $\mathrm{X}$ and $\mathrm{Li} \mathrm{J}$ : Era of universal testing of microsatellite instability in colorectal cancer. World J Gastrointest Oncol 5: 12$19,2013$.

10 Dudley JC, Lin MT, Le DT and Eshleman JR: Microsatellite Instability as a Biomarker for PD-1 Blockade. Clin Cancer Res 22: 813-820, 2016.

11 Le D, Uram J, Wang H, Bartlett B, Kemberling H, Eyring A, Saba Azad N, Laheru D, Ross C. Donehower R, Crocenzi TS, Goldberg RM, Fisher GA, Lee JJ, Greten TF, Koshiji M, Kang SP, Anders RA, Eshleman JR, Vogelstein B and Diaz LA: Programmed death-1 blockade in mismatch repair deficient colorectal cancer. J Clin Oncol 34(suppl): abstract 103, 2016.
12 Overman M, Lonardi S, Leone F, McDermott R, Morse M, Wong K, Neyns B, Leach J, Alfonso PG, Lee JJ, Hill A, Lenz H-J, Desai J, Moss RA, Cao ZA, Ledeine J-M, Tang H, Kopetz $\mathrm{S}$ and Andre T: Nivolumab in patients with DNA mismatch repair deficient/microsatellite instability high metastatic colorectal cancer: Update from CheckMate 142. J Clin Oncol 35(suppl 4S): abstract 519, 2017.

13 National Comprehensive Cancer Network. Colon Cancer (Version 2.2017). https://www.ncen.org/professionals/physician_ gls/pdf/colon.pdf. Accessed April 26, 2017.

14 Achanta CR, Dahiya D, Garg M, Rana SS and Behera A: Education and Imaging. Gastrointestinal: colon cancer with cutaneous infiltration. J Gastroenterol Hepatol 28: 762, 2013.

15 Marcoval J, Moreno A and Peyri J: Cutaneous infiltration by cancer. J Am Acad Dermatol 57: 577-580, 2007.

16 Tenreiro N, Ferreira C, Silva S, Marques R, Ribeiro A, Sousa PJ and Luis FP: Locally advanced colon cancer with cutaneous invasion: case report. BMC Res Notes 10: 113, 2017.

17 Bolandparvaz S, Jelodar S, Heidari Esfahani M and Moslemi S: Chemotherapy-Induced Perforation of Gastric Burkitt Lymphoma; A Case Report and Review of the Literature. Bull Emerg Trauma 2: 133-135, 2014.

18 Neduka H, Munemoto M, Ojima T, Earashi M, Fujii H and Nonomura A: Highly Advanced Gastric Cancer Leading to Immediate Perforation on Day Three of Chemotherapy with S1 plus Oxaliplatin. Gan To Kagaku Ryoho 43: 1939-1941, 2016.

19 Suzaki N, Hiraki A, Ueoka H, Aoe M, Takigawa N, Kishino T, Kiura K, Kanehiro A, Tanimoto M and Harada M: Gastric perforation due to metastasis from adenocarcinoma of the lung. Anticancer Res 22: 1209-1212, 2002.

20 Papavramidis TS, Mantzoukis $\mathrm{K}$ and Michalopoulos N: Confronting gastrocutaneous fistulas. Ann Gastroenterol 24: 1619, 2011.

21 Ahmed F, Goodman MT, Kosary C, Ruiz B, Wu XC, Chen VW and Correa $\mathrm{CN}$ : Excess risk of subsequent primary cancers among colorectal carcinoma survivors, 1975-2001. Cancer 107: 1162-1171, 2006.

22 Bhatia S: Therapy-related myelodysplasia and acute myeloid leukemia. Semin Oncol 40: 666-675, 2013.

23 Vardiman JW, Harris NL and Brunning RD: The World Health Organization (WHO) classification of the myeloid neoplasms. Blood 100: 2292-2302, 2002.

24 Le Roy A, Kempf E, Ackermann F, Routier E, Robert C, Turpin A, Marabelle A, Mateus C, Michot JM and Lambotte O: Two cases of immune thrombocytopenia associated with pembrolizumab. Eur J Cancer 54: 172-174, 2016.

25 Nair R, Gheith S and Nair SG: Immunotherapy-Associated Hemolytic Anemia with Pure Red-Cell Aplasia. N Engl J Med 374: 1096-1097, 2016.

Received May 27, 2017

Revised June 7, 2017

Accepted June 8, 2017 